\title{
Differential locus coeruleus and hypothalamic self-stimulation interactions
}

\author{
RICHARD J. BODNAR \\ New York State Psychiatric Institute, New York, New York 10032 \\ STEVEN J. ELLMAN \\ City College of New York, New York, New York 10033 \\ EDGAR E. COONS \\ New York University, New York, New York 10003 \\ and \\ ROBERT F. ACKERMANN and SOLOMON S. STEINER \\ City College of New York, New York, New York 10033
}

\begin{abstract}
To ascertain the nature of intracranial self-stimulation (ICSS) interactions between locus coeruleus (LC) and various hypothalamic (HYP) sites, rats were tested with monophasic pulse pair stimulation. When trains of pulse pairs were delivered to a single ICSS site, lower responding occurred at short pulse-pair intervals. However, when the pulses of each pulse pair were split between LC and HYP ICSS sites, response rates at all pulse-pair intervals were elevated well above single-pulse responding, indicating a neurophysiological interaction between these loci. The symmetry of LC and HYP interactions depended upon the HYP electrode placement. Both internal capsule and fields of Forel placements produced asymmetrical LC-HYP interactions in which higher ICSS rates occurred when the LC received the first pulse of each pair than when it received the second pulse of each pair, suggesting a direct, and possibly modulating, influence of the LC upon these HYP placements. Both medial forebrain bundle and perifornical HYP placements produced symmetrical LC-HYP interactions in which similar ICSS rates occurred regardless of site stimulation order, suggesting an indirect, reciprocal influence between the LC and these HYP placements.
\end{abstract}

Theories of intracranial self-stimulation (ICSS) behavior have often questioned whether the many discrete neuroanatomical loci throughout the brain which subserve the behavior are linked to form an integrated "reward" system or if these loci are independent of one another and only share the common property of ICSS. Among the many means of ascertaining whether ICSS loci form an integrated system is to determine whether stimulation delivered to pairs of ICSS sites will summate and cause increased ICSS responding. Previous studies demonstrated that response rates elicited under simultaneous or nearsimultaneous stimulation of two ICSS sites were significantly greater than the sum of the response rates elicited when each of the sites was stimulated singly, suggesting that a behavioral interaction between the

Data collection and analysis was supported by a CCNY Faculty Research Award to S.J.E. and a NIDA Grant to S.S.S. Manuscript preparation was supported by N.Y.S. Health Research Council Grant 922 to R.J.B. We thank M. Brutus, J. Healey, P.M. Ippolito, and W.T. Nelson for invaluable technical assistance. Send reprint requests to: R. J. Bodnar, Department of Behavioral Physiology, New York State Psychiatric Institute, 722 West 168th Street, New York, New York 10032. two ICSS sites occurred (Ackermann, Bodnar, Jackler, Steiner, \& Ellman, 1973; Coons, Smith, \& Ungerleider, 1976; Ellman, Ackermann, Bodnar, Jackler, \& Steiner, 1975; Ungerleider \& Coons, 1970). Such ICSS interactions have been demonstrated for the following electrode site combinations: the locus coeruleus (LC) and hypothalamus (HYP), the midventral periaqueductal gray and HYP, the dorsolateral periaqueductal gray and HYP, the substantia nigra and HYP, and bilateral HYP sites (Ackermann et al., 1973; Coons et al., 1976; Ellman et al., 1975; Ungerleider \& Coons, 1970).

A monophasic stimulation technique, initially employed to determine the refractory characteristics of peripheral nervous system neurons (Erlanger \& Gasser, 1937; Lloyd, 1946; Sherrington, 1906), has provided an ideal way to study the magnitude and strength of ICSS behavioral interactions. In a modification of the basic technique for use in ICSS studies, Deutsch (1964) delivered stimulation trains of paired monophasic rectangular electrical pulses to an animal's electrode sites in order to approximate the refractory characteristics of the neurons subserving ICSS behavior. The first pulse of each pair was designated 
the $\mathrm{C}$ pulse and the second pulse designated the $\mathrm{T}$ pulse. The temporal distance between succeeding $C$ pulses in a stimulation train is called the $C-C$ interval, while the temporal distance between $\mathrm{C}$ and $\mathrm{T}$ pulses is called the C-T interval. In a series of studies (Gallistel, 1969; German \& Holloway, 1972; Szabo, Lenard \& Kosaras, 1974; Yeomans, 1975), ICSS response rates were shown to be altered by the temporal spacing of the pulse pairs. When the C-T interval was shortened below a critical value (.5-1.2 $\mathrm{msec})$, ICSS response rates sharply diminished to a value elicited by stimulation comprising only $\mathrm{C}$ pulses ( $\mathrm{T}$ pulses omitted), suggesting that at the very short $\mathrm{C}-\mathrm{T}$ intervals the $\mathrm{T}$ pulses are rendered ineffective. Further studies (Coons et al., 1976; Rolls, 1971c; Smith \& Coons, 1970) demonstrated that the current necessary to support a criterion ICSS rate was significantly increased as the $C$ - $T$ interval was shortened below this same critical value, indicating that the limitations of these intervals were not response dependent. Moreover, the relationship between C-T interval and ICSS behavior can only subtly be modified by alterations in both anodal and cathodal loci (Bodnar, Steiner, Brutus, Ippolito, \& Ellman, 1978; Bodnar, Steiner, Healey, Halperin, \& Ellman, 1978).

The inability of a single ICSS site to sustain peak responding at short $\mathrm{C}-\mathrm{T}$ intervals can serve as a reliable marker for the determination of the existence of an interaction between two ICSS sites, as demonstrated initially by Ungerleider and Coons (1970). When the monophasic trains of stimulation were divided between bilateral medial forebrain bundle (MFB) electrode placements such that the $C$ pulses were delivered to one side while the $\mathrm{T}$ pulses were delivered to the contralateral side, criterion response rates were achieved at the same current intensity across all C-T intervals, including those $(.5-1.2 \mathrm{msec})$ which failed to support these response levels when both pulses of the pulse pair were delivered to the same site (Coons et al., 1976; Ungerleider \& Coons, 1970). These data suggest that the stimulation effects at both bilateral MFB ICSS sites were summating through either temporal or spatial convergence. German and Holloway (1973) revealed that when the $\mathrm{C}$ and $\mathrm{T}$ pulses were divided between asymmetrical bilateral HYP ICSS electrode placements (preoptic HYP and posterior MFB), interaction response rates were greater when the $C$ pulses were delivered to the preoptic HYP and the T pulses to the posterior MFB than when the $C$ pulses were delivered to the posterior MFB and the T pulses to the preoptic HYP. To these investigators, the higher responding for the C-preoptic HYP, T-posterior MFB condition implied that the structures responsible for the summating interaction were more distant from the preoptic HYP than from the posterior MFB, assuming: (a) that the highest response rate would be contingent upon the simultaneous arrival of a neural excitation caused by the stimulation at the summation point, and (b) that this summation point must be more distant from the preoptic HYP than from the posterior MFB given that the highest rates occur when the preoptic HYP receives the earlier pulse of each pair (German \& Holloway, 1973).

The present study applied the same techniques and reasoning to the study of ICSS interactions between the locus coeruleus (LC), a pontine nuclear cell group (Ungerstedt, 1971), which supports ICSS behavior (Crow, Spear, \& Arbuthnott, 1972; Ellman, Ackermann, Farber, Mattiace, \& Steiner, 1974; Ritter \& Stein, 1973) and various HYP loci. A recent study (Farber, Ellman, Mattiace, Holtzman, Ippolito, Halperin, \& Steiner, 1976) showed that unilateral LC lesions selectively reduced or abolished ICSS elicited from ipsilateral crus cerebri or fields of Forel HYP electrode placements, while sparing or facilitating MFB or perifornical ICSS. This latter result has also been reported elsewhere (Koob, Balcom, $\&$ Meyerhoff, 1976). The present study investigated whether the monophasic pulse-pair interaction technique would be capable of differentiating LC-HYP interactions as a function of HYP locus. Specifically, we predicted that higher ICSS response rates would occur when the $C$ pulses were delivered to the LC and the $T$ pulses to the crus cerebri or fields of Forel than when the reverse sequence was tested. The rationale for this prediction was that since LC lesions reduce or abolish crus cerebri/fields of Forel ICSS, interaction response rates should be higher when the LC was stimulated first, because activation of the LC is a necessary condition either for activation at the crus cerebri/fields of Forel themselves or for activation at some other locus at which the LC and crus cerebri/fields of Forel neurons interact. In contrast, those HYP loci unaffected by LC lesions (MFB and perifornical area) should fail to display such interaction response-rate asymmetries.

\section{METHOD}

Twelve male Sprague-Dawley albino rats (375-500 g) were anesthetized with Chloropent (Fort Dodge Laboratories; $1 \mathrm{ml} / \mathrm{kg}$ ) and stereotaxically (Kopf) implanted with two stainless steel screws and two stainless steel bipolar electrodes (MS 301/1, Plastic Products) aimed ipsilaterally at the LC and HYP. The bipolar electrodes were twisted wires, $.35 \mathrm{~mm}$ in outside diameter, and were completely insulated except at the tips. The electrode tips were aligned mediolaterally so as to maximize stimulation of longitudinally running fibers (Szabo \& Milner, 1973). The two steel screws were screwed into the skull and electrically connected to an electrode plug. With the incisor bar set at $-5 \mathrm{~mm}$, LC electrode coordinates were $1.5-2.0 \mathrm{~mm}$ posterior to lambda, $1.0 \mathrm{~mm}$ lateral to the sagittal suture, and $7.0 \mathrm{~mm}$ from the top of the skull, while HYP electrode coordinates were 4.2-4.4 mm posterior to bregma, $1.5 \mathrm{~mm}$ lateral to the sagittal suture, and $8.7 \mathrm{~mm}$ from the top of the skull. 
Ten days after surgery, each animal was shaped to barpress for negatively going, capacitance-coupled, monophasic rectangular pulses of electrical brain stimulation to each electrode site in an operant conditioning chamber on a continuous reinforcement schedule. Any responses made during a stimulation train were ineffective. The stimulator was constructed from Digi-Bit solidstate logic circuitry (BRS/LVE) which allowed the experimenter to set the pulse duration and the C-C interval and independently manipulate whether or not the $T$ pulses were delivered, and if delivered, the C-T interval. Waveform, C-C interval, C-T interval, stimulus intensity, and pulse and train duration were continuously monitored by observing on a cathode ray oscilloscope the voltage drop across a 100-ohm resistor in series with the animal.

During preliminary screening, the animals were shaped for a maximum of 15 daily sessions at each electrode site to barpress for 700 -msec trains of electrical stimulation at a frequency of 200 pulses/sec and a pulse duration of $.1 \mathrm{msec}$. The pole of each bipolar electrode which elicited the higher peak response rates at the lowest intensity in this preliminary screen (Steiner, Bodnar, Nelson, Ackermann, \& Ellman, 1978) served as the cathode for the remainder of the experiment and either the other pole of the same bipolar electrode or a cortical screw served as the anode. For each electrode site, the lowest intensity was chosen which yielded consistently high response rates at a C-C interval of 10.0 msec and a C-T interval of $5.0 \mathrm{msec}$, but only operant responding if the $T$ pulses were omitted. The criterion intensities across animals varied from 200 to $600 \mu \mathrm{A}$, comparable to or lower than intensities used in other studies employing monophasic pulse pairs of brief .1 msec duration (Bodnar, Steiner, Brutus, et al., 1978; Bodnar, Steiner, Healey, et al., 1978; Deutsch, 1964; Gallistel, 1969; German \& Holloway, 1972; Rolls, 1971c; Smith \& Coons, 1970; Szabo et al., 1974). The number of microcoulombs (stimulation delivered per unit time) delivered in this stimulation technique is substantially less than, that delivered by traditional $60-\mathrm{Hz}$ sinusoidal stimulation.

After preliminary testing, the stimulation parameters were altered such that each animal barpressed at each of its electrode sites at a C-C interval of $30 \mathrm{msec}$. Following this, each rat's electrode sites were individually tested during a 72-min session which was divided into nine 7 -min periods. A 10 -min time-out occurred between each period, during which the C-T interval was changed. Eight C-T intervals $(.5, .8,1.0,1.2,1.5,2.0,3.0,5.0 \mathrm{msec})$ and a condition in which the $T$ pulses were omitted were randomly presented in each of 9 days in a Latin square design. The mean response rate over the last $5 \mathrm{~min}$ of each 7 -min period constituted the dependent variable. Data from the first 2 min of each 7 -min period were excluded from the analysis of experimental effects but revealed that the Latin square design adequately controlled for order and carry-over effects. The Latin square was constructed such that (a) each stimulation condition occupied each ordinal position of the square only once over the 9-day procedure, and (b) each stimulation condition preceded each other stimulation condition only once over the 9-day procedure. C-T interval/ response rate functions, averaged over the 9-day paradigm, were determined for both LC and HYP sites in each animal.

After these functions were established for each site, each animal was retested under the same conditions (C-C interval, $30 \mathrm{msec}$; pulse duration, $.1 \mathrm{msec}$; train duration, $700 \mathrm{msec}$ ), with the exception that the $C$ and $T$ pulses were split between the two electrode sites and delivered at each site's respective current intensity. In one condition, the $\mathrm{C}$ pulses were delivered to the $\mathrm{LC}$ at its current intensity and the $\mathrm{T}$ pulses were delivered to the HYP at its current intensity. In the reverse condition, the C pulses were delivered to the HYP at its current intensity, and the $\mathrm{T}$ pulses were delivered to the $\mathrm{LC}$ at its current intensity. Nine C-T intervals, randomly presented in a Latin square design were tested each day for 18 days. Nine days of C-LC, T-HYP data and 9 days of C-HYP, T-LC data, either condition alternating over days in an a-b-b-a manner, were collected across the nine C-T intervals.
After completion of the experiment, all animals were overdosed with Chloropent and perfused through the heart with normal saline followed by $10 \%$ Formalin. Serial frozen sections were stained with luxol fast blue and cresyl violet (Klüver \& Barrera, 1953), and electrode loci were determined by microscopic examination of the sections. All localizations were carried out by a rater who was uninformed with respect to each animal's behavioral data. Animals were placed into one of two groups on the basis of the location of their HYP electrode. The first group comprised those HYP sites (crus cerebri/fields of Forel) which showed reduced ICSS response rates following LC lesions (Farber et al., 1976). The second group comprised those HYP sites (MFB/periforical area) which showed unaltered ICSS response rates following LC lesions (Farber et al., 1976).

\section{RESULTS}

All 12 animals supported ICSS behavior from both LC and HYP electrodes at criterion levels, that is, the chosen intensity for each site supported consistently high (range: 8-120 responses/min) response rates at a C-T interval of $5 \mathrm{msec}$, yet supported response rates that were always significantly less than rates at a C-T interval of $5 \mathrm{msec}$ when the $\mathrm{T}$ pulses were omitted (correlated difference score $t$ test, $t(11)=$ $10.06, p<.01)$. Each site in each animal yielded similar ICSS behavior, since no significant differences were observed across electrode sites either in the operant ICSS response rates elicited when the $T$ pulses were omitted or in the criterion intensities employed. In all animals, the tip of the posterior electrode employed as the cathodal source during behavioral testing was located in either the LC proper or its ascending dorsal noradrenergic bundle. In 4 animals, the tip of the HYP electrodes employed as the cathodal source during behavioral testing impinged upon either the internal capsule at its interface with the far-lateral HYP or the fields of Forel hereafter designated the dorsal or far-lateral hypothalamus (DFLH); these animals comprised the LC-DFLH group. Figure 1 (Rat 67G) shows a representative animal in the LC/DFLH group; the cathodal sources for both electrodes were the lateral tips. In 8 animals, the tip of the HYP electrodes employed as the cathodal source during behavioral testing impinged upon either the MFB or the perifornical area; these animals comprised the LC-MFB group. Figure 1 (Rat 61G) shows a representative animal in the LC-MFB group. The cathodal source of the LC electrode was the lateral tip, while the cathodal source of the HYP electrode was the medial tip. As can be seen, the LC placements are very similar to those of the other group leaving only the HYP placements differentiated. Figures 2 and 3 exhibit the precise electrode localizations of each tip employed as the cathodal source for each electrode site for each animal in each group.

Figures $4 \mathrm{a}$ and $4 \mathrm{~b}$ display representative C-T interval/response rate functions for LC and HYP 

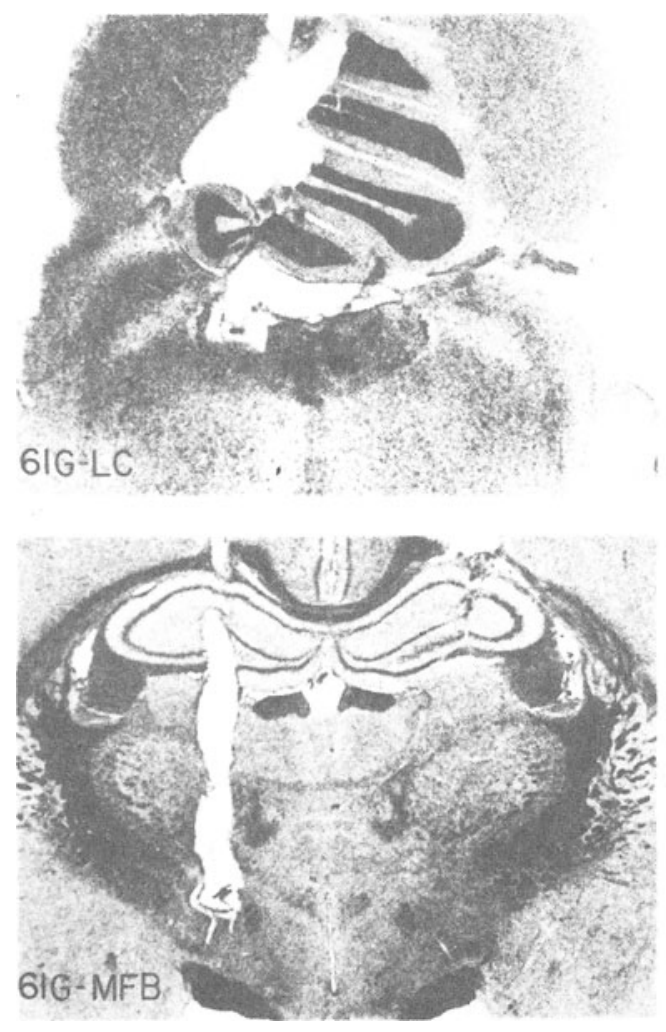
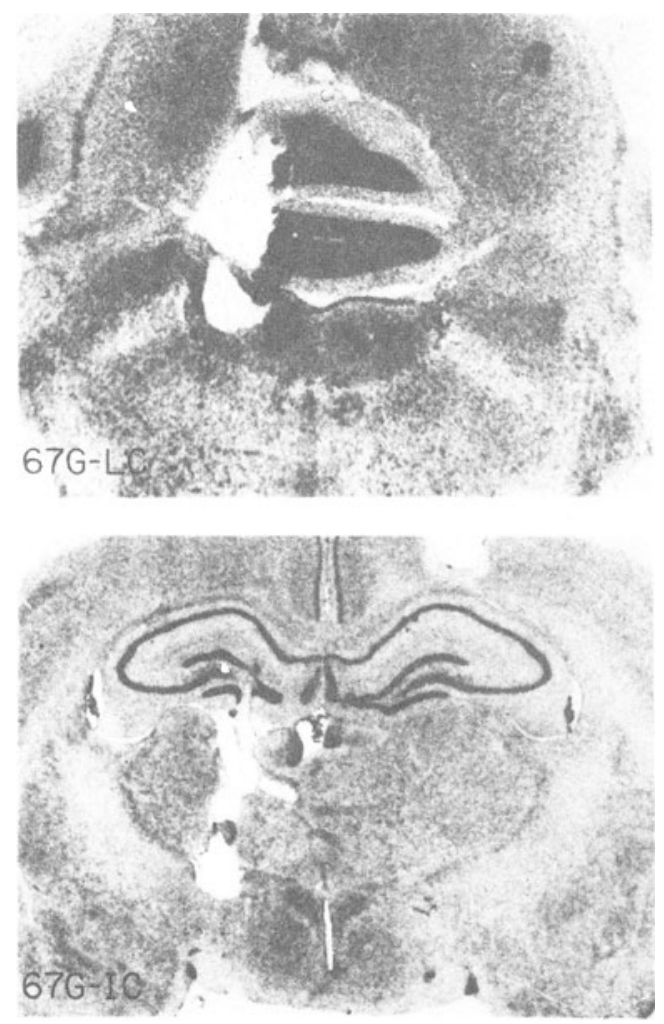

Figure 1. Photomicrographs of coronal sections showing the locus of electrode placements for two representative animals. The sections on the left display the placements of an animal in the LC-MFB group. The cathodal source of the LC electrode was the lateral tip, while the cathodal source of the HYP electrode was the medial tip. Symmetrical interaction response rates were observed for the C-LC, T-MFB and the C-MFB, T-LC conditions. The sections on the right display the placements of an animal in the LC-DFLH group; the cathodal sources for both electrodes were the lateral tips. Asymmetrical interaction response rates in which the C-LC, T-DFLH condition produced higher responding than the C-DFLH, T-LC condition were observed. Note that the potine electrode placements are almost identical for both groups.

electrode sites, respectively, for the experimental conditions under which the $\mathrm{C}$ and $\mathrm{T}$ pulses were delivered to the same site. One-way analyses of variance for each electrode site in each animal in each group revealed that at least one C-T interval, usually between .5 and $1.0 \mathrm{msec}$, in each function displayed ICSS response rates which were significantly lower than those elicited at a C-T interval of $5.0 \mathrm{msec}$ yet similar to those elicited when the $\mathrm{T}$ pulses were omitted. As the C-T interval was lengthened, ICSS response rates typically recovered to near peak $(1.2$ to $1.5 \mathrm{msec})$ and then peak $(2.0$ to $3.0 \mathrm{msec}) \mathrm{re}$ sponding for each electrode site in each group (Bodnar, Steiner, Healey, et al., 1978).

A three-way analysis of variance was performed on the interaction data of all 12 rats which analyzed the experimental groups (LC-DFLH and LC-MFB) as one main effect, the site stimulation order (C-LC, T-HYP and C-HYP, T-LC) as a second main effect, and the contribution of $\mathrm{T}$ pulses at each C-T interval as the third main effect. First, no significant dif- ferences $[F(8,180)=.04]$ among the response rates elicited at each C-T interval were noted. Thus, unlike the single-site conditions which exhibited significant decrements at short $(.5-1.5 \mathrm{msec})$ as compared to longer $(1.5-5.0 \mathrm{msec}) \mathrm{C}-\mathrm{T}$ intervals, interaction response rates remained constant across all intervals. Moreover, for both stimulation conditions in both groups, response rates at each $\mathrm{C}$ - $\mathrm{T}$ interval were always significantly (ANOVAs, $p<.05$ ) greater than the sum of response rates when only $C$ pulses were delivered to each site. Figure $4 c$ displays a representative $C-T$ interval response rate function when the $C$ and $T$ pulses were split between the two electrode sites.

Second, no significant differences in interaction response rates were observed $[\mathrm{F}(1,180)=1.48]$ between the LC-DFLH $(X=43.8)$ and the LC-MFB $(\mathrm{X}=40.8)$ groups, indicating that both groups produced similar response magnitudes in the interaction. Third, the C-LC, T-HYP $(\mathrm{X}=44.8)$ condition yielded significantly higher $[\mathrm{F}(1,80)=6.80, \mathrm{p}<.05]$ 


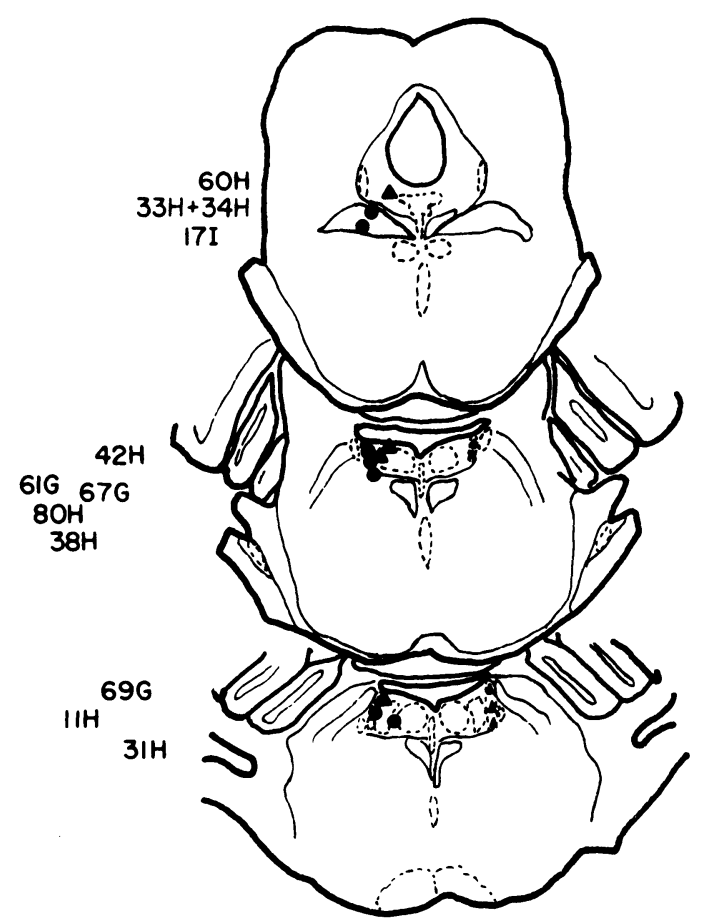

$3 a$

Figure 2. Schematic representation (Palkovits \& Jacobowitz, 1974) of the locus coeruleus placements for both the LC-DFLH (triangles) and LC-MFB (circles) groups.

interaction response rates than its C-HYP, T-LC $(\mathrm{X}=38.8)$ counterpart. Moreover, a posteriori Scheffé comparisons revealed that this effect could be accounted for by the performance of the LC-DFLH group rather than the LC-MFB group.

For the four animals in the LC-DFLH group, the C-LC, T-DFLH condition ( $\mathrm{X}=53.1)$ yielded significantly higher $[F(1,70)=14.32, p<.01]$ interaction response rates than its C-DFLH, T-LC counterpart $(X=34.5)$. Figure 5 displays this relationship for all four animals in the LC/DFLH group. As can be seen, no overlap in response rate between the two conditions at any C-T intervals was observed in any animal in this group.

For the eight animals in the LC-MFB group, no significant differences $[F(1,142)=.15]$ in interaction response rates were observed between the $C-L C$, T-MFB ( $X=40.6)$ and the C-MFB, T-LC (X = 40.9) conditions. Figure 6 displays this relationship for four of the eight animals in this group. Unlike the LC-DFLH group, in which no overlap in response rate occurred for any animal between C-LC, T-HYP and C-HYP, T-LC conditions over C-T intervals, the LC-MFB group displayed overlaps in responding between the two conditions at each C-T interval. This pattern even persisted in the single LC-MFB animal (Rat $11 \mathrm{H}$, Figure 6), which displayed overall significantly differential responding.

\section{DISCUSSION}

The present study demonstrated that ICSS interactions occur between LC and various HYP ICSS sites and that the nature of such interactions in terms of ICSS responding depends upon the locus of the HYP electrode placement. These differences seem to be site dependent and not attributable to other factors. First, for all LC and HYP electrode placements of both groups, response rates elicited at short $\mathrm{C}-\mathrm{T}$ intervals when $\mathrm{C}$ and $\mathrm{T}$ pulses were delivered to the same site were comparable to those elicited when the $T$ pulses were omitted and significantly lower than rates elicited at longer C-T intervals. These results are in accord with those reported previously (Bodnar, Steiner, Brutus, et al., 1978; Bodnar, Steiner, Healey, et al., 1978; Deutsch, 1964; Gallistel, 1969; German \& Holloway, 1972; Rolls, 1971c; Smith \& Coons, 1970; Szabo et al., 1974; Yeomans, 1975). ICSS response rates when the $\mathrm{T}$ pulses were omitted did not differ across electrode sites or across groups, nor did the criterion inten-

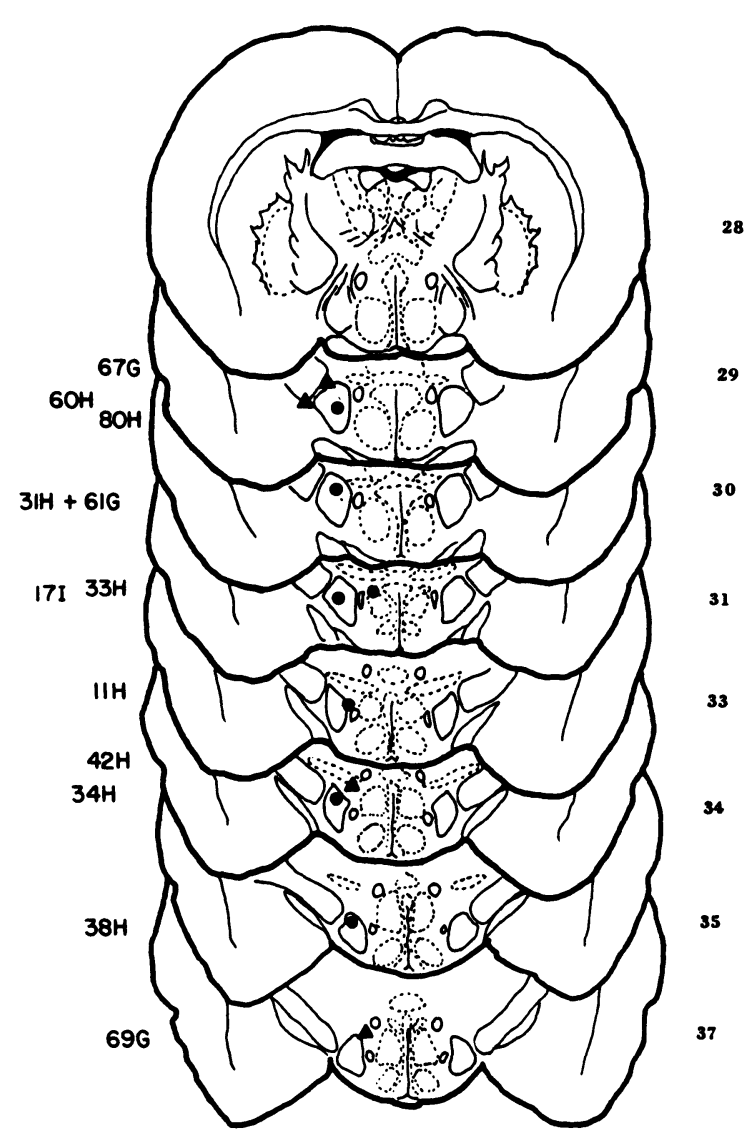

Figure 3. Schematic representation (König \& Klippel, 1963) of the hypothalamic placements for both the LC-DFLH (triangles) and LC-MFB (circles) groups. 
A

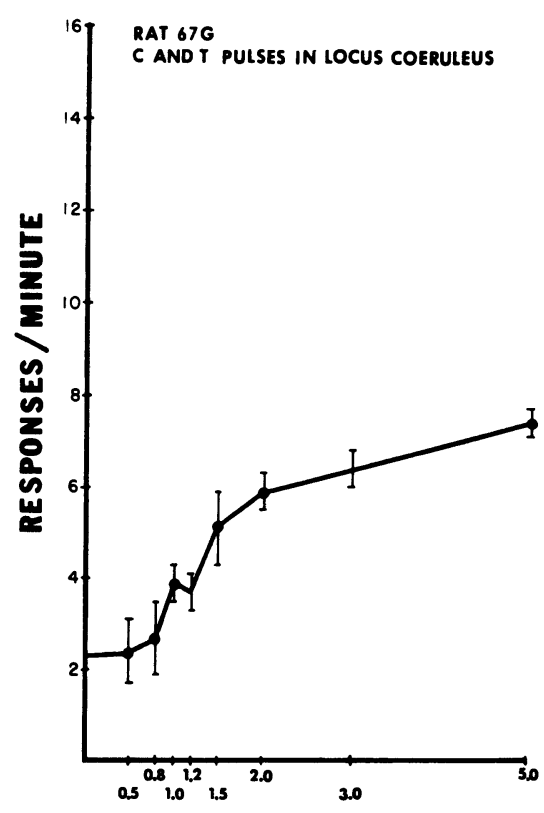

B

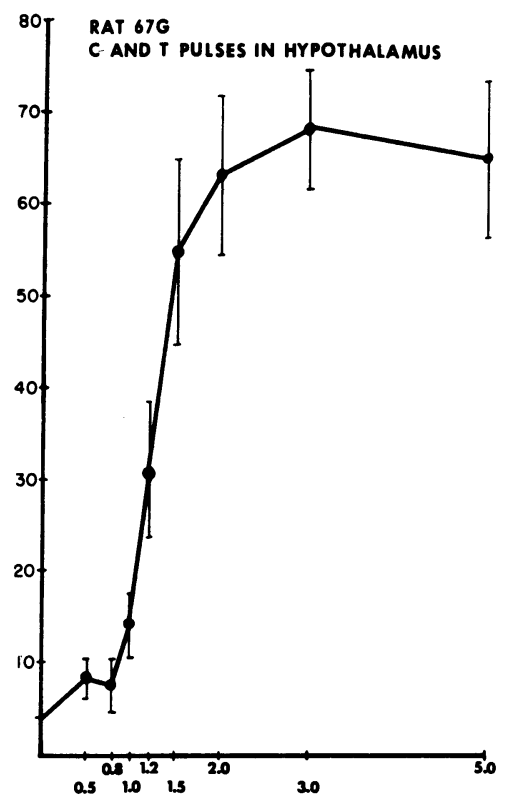

C

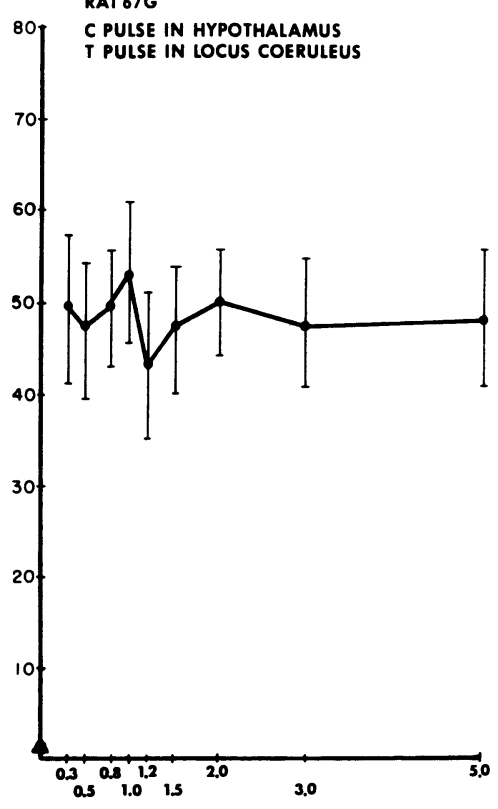

C-T INTERVAL(MSEC)

Figure 4. (a) ICSS response rate $( \pm$ SEM) as a function of C-T interval for a representative LC placement when both $C$ and $T$ pulses were delivered to the same site. (b) ICSS response rate $( \pm S E M)$ as a function of $C$ - $T$ interval for a representative HYP placement when both $C$ and $T$ pulses were delivered to the same site. $C-C$ performance alone is depicted the ordinate axis for $a$ and $b$. (c) ICSS interaction response rate $( \pm S E M)$ as a function of C-T interval for a representative animal when the $C$ pulses were delivered to the $L C$ and the $T$ pulses to the HYP. The triangle on the ordinate represents the sum of response rates elicited when $\mathbf{C}$ pulses were delivered to each site singly.

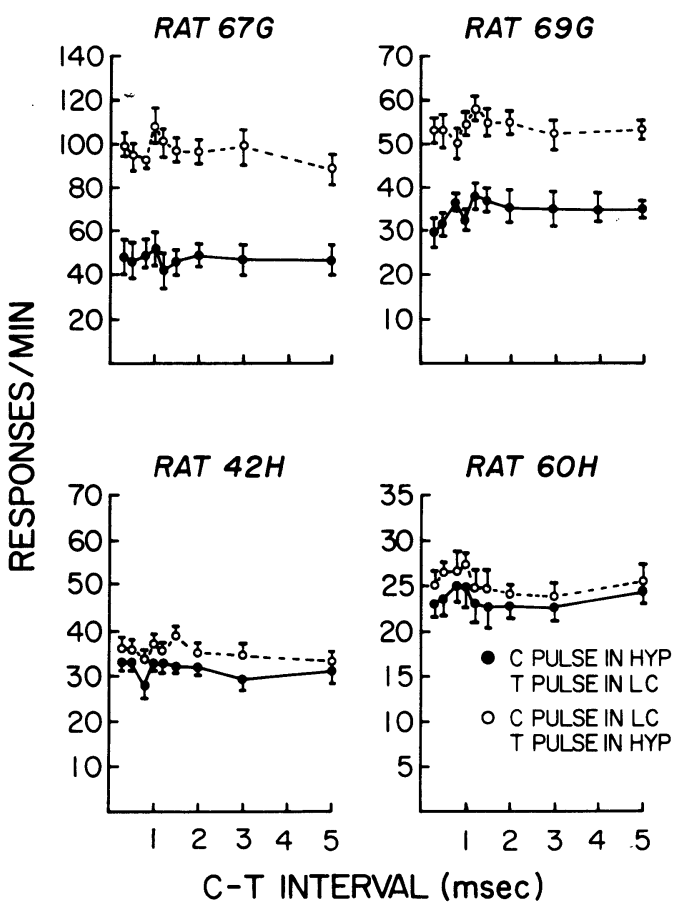

Figure 5. ICSS interaction response rate $( \pm$ SEM) as a function of $C$-T interval and as a function of which site receives the $C$ and $T$ pulse for the four animals in the LC-DFLH group. Note the nonoverlapping response rate asymmetries typical of all animals in this group.

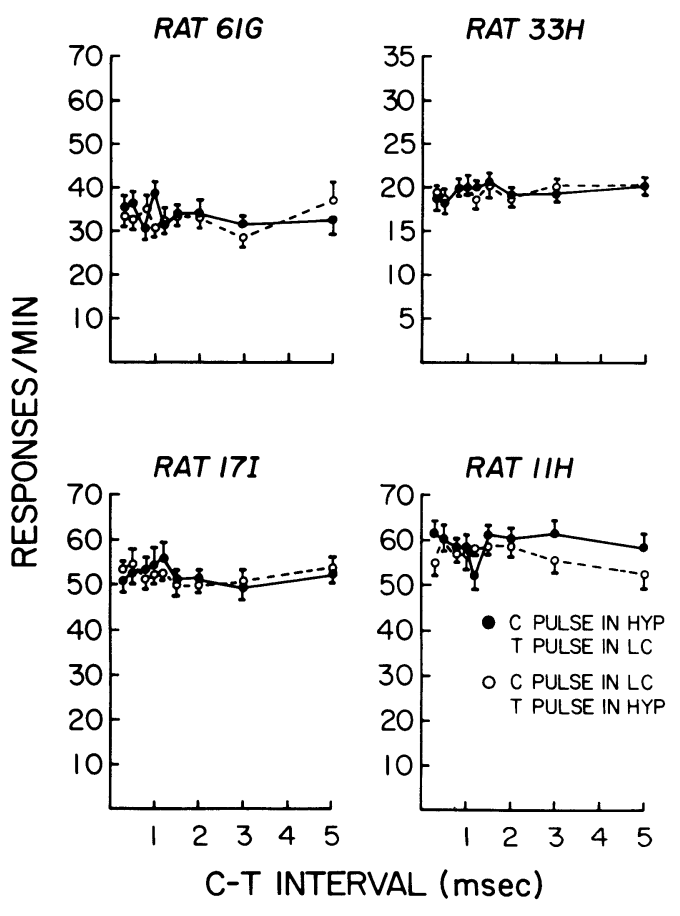

Figure 6. ICSS interaction response rate $( \pm$ SEM) data for four animals in the LC-MFB group. Note the overlapping response rate symmetry typical of seven or eight animals in this group. The one rat $(11 \mathrm{H})$ that displayed complementary response rate asymmetry to that of the DFLH group also did overlap across intervals. 
sity employed differ. Second, all animals in all groups displayed a behavioral interaction, since, in all cases when pulse pairs were split between the two ICSS electrode sites, interaction response rates were significantly greater than the sum of response rates when only $\mathrm{C}$ pulses were delivered to each site. This was true at all C-T intervals tested and regardless of which site was stimulated first. These results are also in accord with previously published data (Ackermann et al., 1973; Coons et al., 1976; Ellman et al., 1975; German \& Holloway, 1973; Ungerleider \& Coons, 1970) and suggest that the LC and HYP interact neurophysiologically. Third, these differences, and indeed the interactions themselves, cannot be attributable to such nonspecific factors as passive current spread, since the monophasic pulse pair technique was employed in the present study. Stimulation consists of brief .1-msec-duration square-wave pulses the cathodal influence of which almost instantaneously dissipates at the end of the pulse delivery. The interaction parameters employed in the present study are such that pulses delivered to the two sites were separated by a minimum interval of $.3 \mathrm{msec}$. Given the instantaneous dissipation rate of monophasic square-wave stimulation, it is inconceivable that passive spread of current produced by the $\mathrm{C}$ pulses delivered at one electrode site can affect the cathodal properties of the $\mathrm{T}$ pulses delivered at a second electrode site. Furthermore, if current spread produced the interaction, it still could not explain why response rate asymmetries which are solely dependent upon the order of stimulus presentation at each electrode site exist in the LC-DFLH group, but not in the LC-MFB group. It is apparent that these results can only be interpreted in terms of differential neurophysiological interactions between ICSS sites.

The symmetry or asymmetry of the ICSS interactions between the LC and HYP was dependent upon the location of the HYP placement. If a DFLH electrode was employed,the interaction was asymmetrical in that interaction response rates were significantly greater for the C-LC, T-DFLH condition than for the C-DFLH, $\mathrm{T}$ - $\mathrm{LC}$ condition across all C-T intervals. If it is assumed that high response rates are a product of simultaneous or near-simultaneous arrival of the neurophysiological consequences of stimulating the two electrode sites at some common summation point. The implication would be that this point would be located neurophysiologically more distant from the LC than from the DFLH electrode sites. If the summating point is either the DFLH itself or some third site, then it would be possible for the neurophysiological consequences of stimulating the LC and DFLH to arrive simultaneously at the summating point only if the $\mathrm{LC}$ receives the initial pulses. In contrast, if the $C$ pulses are delivered to the DFLH, then the neurophysiological consequences of LC stimulation would always arrive at the summating point after that of DFLH. In contrast, if a MFB HYP electrode was employed, the interaction was symmetrical in the C-LC, T-MFB condition and the C-MFB, T-LC condition interaction response rates were similar. The only exception was an asymmetrical interaction which was the reverse of the DFLH group, and which was limited to only some C-T intervals. These data imply either a reciprocity of connections involved in LC-MFB interactions or that the summation point is neurophysiologically equidistant from the LC and MFB.

Unit recording of brainstem loci, including the area of the midbrain LC and its ascending dorsal noradrenergic bundle, has been shown to be directly activated by hypothalamic stimulation. When pulse pairs were delivered to hypothalamic sites at intervals less than .8-1.1 msec, unit action potentials were recorded for the initial but not the trailing pulses of the pair while at intervals greater than $1.1 \mathrm{msec}$, unit action potentials were recorded for both pulses of the pair (Gallistel, Rolls \& Greene, 1969; Matthews \& Gallistel, 1975; Rolls, 1971a, 1971b, 1971c). Such data correspond with the onset of peak ICSS responding at the longer C-T intervals and with the diminution in response rates at shorter C-T intervals (Bodnar, Steiner, Brutus, et al., 1978; Bodnar, Steiner, Healey, et al., 1978; Deutsch, 1964; Gallistel, 1969; German \& Holloway, 1972; Rolls, 1971c; Smith \& Coons, 1970; Szabo, 1974; Yeomans, 1975).

An ascending directionality hypothesis would predict higher response rates when the LC receives the earlier pulse of each pair than when it would receive the later pulse, since the neurophysiological consequences of LC stimulation would take longer to reach the summating forebrain structures than from the DFLH. A second explanation of the results could involve the lifetime of the released transmitter at the convergent site of LC and DFLH stimulation. If the neurophysiological consequences of LC stimulation released a "long-lived" transmitter while DFLH stimulation released "short-lived" transmitters, then initial activation of the LC would leave summative and convergent capability for the shorter acting DFLH. On the other hand, if the DFLH received the earlier pulses, then the summative characteristics should be less, since the "short-lived" DFLH transmitter would be partially dissipated by the time the effect of the "long-lived" LC transmitter arrived. A third explanation would involve "reverberating circuits" (Hebb, 1949) in which the LC would possess more reverbatory loops than the DLFH, allowing the effects of the LC stimulation to outlast DFLH stimulation. If the $L C$ received the earlier pulse of each pulse pair, the longer activity of the LC would have a greater probability of summating with DFLH activity than if the reverse were tested. For these latter two hypotheses, the 
MFB and LC must necessarily share common characteristics given the symmetry of LC-MFB interactions. Pharmacological data support this notion, since d- but not l-amphetamine enhances both LC and MFB at low doses (Ellman et al., 1975; Ellman, Ackermann, Bodnar, Jackler, \& Steiner, 1976; Phillips, Brooke, \& Fibiger, 1975; Phillips \& Fibiger, 1973; Stephens \& Herberg, 1975), while both isomers nearly equipotently enhance DFLH ICSS at the same doses (Stephens \& Herberg, 1975).

The present data suggest that the LC exerts a potentially modulating influence upon DFLH, but not MFB, ICSS. If initial stimulation of the LC is necessary for optimal summation in ICSS interactions, this suggests that tonic LC activation may be necessary for DFLH ICSS and explain the reduction or abolition of DFLH following LC lesions (Farber et al., 1976). The complement of such an explanation is feasible, since the LC and MFB produce reciprocal and similar interactions, while $\mathrm{LC}$ lesions do not attenuate MFB ICSS (Farber et al., 1976; Koob et al., 1976). Indeed, the occasional asymmetry in which response rates are higher in LC-MFB interactions when the MFB is stimulated initially agrees with the observations that MFB lesions attenuate dorsal noradrenergic bundle ICSS (Clavier $\&$ Routtenberg, 1976). The use of the monophasic pulse pair technique in interaction studies provides an ideal way to determine the complex and heterogeneous mechanisms subserving HYP ICSS and its relationship to other brainstem ICSS sites.

\section{REFERENCES}

Ackermann, R. F., Bodnar, R. J., Jackle R, F., Steine R, S. S., \& Ellman, S. J. Behavioral evidence for neurophysiological interactions between the lateral hypothalamus and locus coeruleus. Proceedings of the Annual Convention of the American Psychological Association, 1973.

Bodnar, R. J., Steiner, S. S., Brutus, M., Ippolito, P. M., \& Ellman, S. J. Hypothalamic self-stimulation differs as a function of anodal locus. Physiological Psychology, 1978, 6, 48-52.

Bodnar, R. J., Steiner, S. S., Healey, J. M., Halperin, J., \& Ellman, S. J. Monophasic pulse pair analysis of intracranial self-stimulation loci. Physiological Psychology, 1978, 6, 170-178.

Clavier, R. M., \& Routtenberg, A. Brainstem self-stimulation attenuated by lesions of medial forebrain bundle but not by lesions of locus coeruleus or the caudal norepinephrine bundle. Brain Research, 1976, 101, 251-271.

Coons, E. E., Smith, N., \& Ungerleider, L. G. Uses of doublepulse stimulation behaviorally to infer refractoriness, summation convergence and transmitter characteristics of hypothalamic reward systems. Journal of Comparative and Physiological Psychology, 1976, 90, 317-342.

Crow, T. J., Spear, P. J., \& Arbuthnott, G. W. Intracranial self-stimulation with electrodes in the region of the locus coeruleus. Brain Research, 1972, 36, 275-287.

DEUTSCH, J. A. Behavioral measurement of the neural refractory period and its application to intracranial self-stimulation. Journal of Comparative and Physiological Psychology, 1964, 58, 1-9.

Ellman, S. J., Ackermann, R. F., Bodnar, R. J., Jackler, F., \& Steiner, S. S. Comparison of behaviors elicited by electrical brain stimulation in dorsal brainstem and hypo- thalamus of rats. Journal of Comparative and Physiological Psychology, 1975, 88, 816-828.

Ellman, S. J., Ackermann, R. F., Bodnar, R. J., Jackler, F., \& STEINER, S. S. D- and l-amphetamine differentially mediate self-stimulation in rat dorsal midbrain area. Physiology \& Behavior, 1976, 16, 1-8.

Ellman, S. J., Ackermann, R. F., Farber, J., Mattiace, L., \& Steiner, S. S. Relationship between dorsal brainstem sleep sites and intracranial self-stimulation. Physiological Psychology, 1974, 2, 31-34.

Erlange R, J., \& Gasser, H. S. Electrical signs of nervous activity. London: Oxford University Press, 1937.

Farber, J., Ellman, S. J., Mattiace, L. A., Holtzman, A., Ippolito, P., Halperin, R., \& Steiner, S. S. Differential effects of unilateral dorsal hindbrain lesions on hypothalamic self-stimulation in the rat. Brain Research, 1976, 112, 148-155.

Gallistel, C. R. The incentive of brain-stimulation reward. Journal of Comparative and Physiological Psychology, 1969, 69, 713-721.

Gallistel, C. R., Rolls, E. T., \& Greene, D. Neuron function inferred from behavioral and electrophysiological estimates of refractory period. Science, 1969, 166, 1028-1030.

German, D. C., \& Holloway, F. A. Behaviorally determined neurophysiological properties of MFB self-stimulation fibers. Physiology \& Behavior, 1972, 9, 823-829.

German, D. C., \& Holloway, F. A. Directionality of rewarding impulses within the medial forebrain bundle self-stimulation system of the rat. Science, 1973, 179, 1345-1347.

HebB, D. O. Organization of behavior. New York: Wiley, 1949.

KLÜver, H., \& BARRERA, E. A method for combined staining of cells and fibers in the nervous system. Journal of Neuropathology and Experimental Neurology, 1953, 12, 400-403.

König, J. F. R., \& KlipPel, R. A. The rat brain. A stereotaxic atlas. Baltimore: Williams and Wilkins, 1963.

Коов, G. F., Balсом, G. J., \& Meyerhoff, J. L. Increases in intracranial self-stimulation in the posterior hypothalamus following unilateral lesions in the locus coeruleus. Brain Research, 1976, 101, 554-560.

LLOYD, D. P. C. Facilitation and inhibition of spinal motorneurons. Journal of Neurophysiology, 1946, 9, 421-438.

Matthews, G., \& Gallistel, C. R. Bilateral interactions in single units driven by MFB self-stimulation electrodes. Physiology \& Behavior, 1975, 15, 543-549.

Palkovits, M., \& J Acobowitz, D. M. Topographic atlas of catecholamine and acetylcholinesterase-containing neurons in the rat brain. II. Hindbrain (mesencephalon, rhombencephalon). Journal of Comparative Neurology, 1974, 157, 29-42.

Phillips, A. G., Brooke, S. M., \& Fibiger, H. C. Effects of amphetamine isomers and neuroleptics on self-stimulation from the nucleus accumbens and dorsal noradrenergic bundle. Brain Research, 1975, 85, 13-22.

Phillips, A. G., \& Fibiger, H. C. Dopaminergic and noradrenergic substates of positive reinforcement: Differential effects of d-and l-amphetamine. Science, 1973, 179, 575-577.

RitTer, S., \& STE in, L. Self-stimulation of noradrenergic cell group (A6) in locus coeruleus of rats. Journal of Comparative and Physiological Psychology, 1973, 85, 443-452.

RoLLS, E. T. Involvement of brainstem units in medial forebrain bundle self-stimulation. Physiology \& Behavior, 1971, 7, 297-310. (a)

Rolls, E. T. Contrasting effects of hypothalamic and nucleus accumbens septi self-stimulation on brainstem single unit activity and cortical arousal. Brain Research, 1971, 31, 275-285. (b)

Rolls, E. T. Absolute refractory period of neurons involved in MFB self-stimulation. Physiology \& Behavior, 1971, 7, 311-315. (c)

She RRIngton, C. The integrative action of the nervous system. New Haven: Yale University Press, 1906.

Smith, N., \& Coons, E. E. Temporal summation and refractoriness in hypothalamic reward neurons as measured by selfstimulation behavior. Science, 1970, 169, 782-785. 
Steiner, S. S., Bodnar, R. J., Nelson, W. T., Ackermann, R. F., \& Ellman, S. J. Intracranial self-stimulation site specificity: The myth of current spread. Brain Research Bulletin, 1978, 3, 349-356.

Stephens, D. N., \& Herberg, L. J. Cathecholamines and selfstimulation: Pharmacological differences between near- and farlateral hypothalamic sites. Brain Research, 1975, 90, 348-351.

Szabo, I., Lenard, L., \& Korsaras, B. Drive decay theory in self-stimulation: Refractory periods and axon diameters in hypothalamic reward loci. Physiology \& Behavior, 1974, 12, 329-343.

Szabo, I., \& Milner, P. M. Electrode tip alignment and selfstimulation; influence of anodal hyperpolarization. Physiology \& Behavior, 1973, 11, 581-583.
Ungerleider, L., \& Coons, E. E. A behavioral measure of homosynaptic and heterosynaptic temporal summation in the self-stimulation of rats. Science, 1970, 169, 785-787.

UNGERSTEDT, U. Stereotaxic mapping of monoamine pathways of the rat brain. Acta Physiological Scandinavica, 1971, Supplement 367, 1-48.

YeOMANS, J. S. Quantitative measurement of neural poststimulation excitability with behavioral methods. Physiology \& Behavior, 1975, 15, 593-602.

(Received for publication April 27, 1979; revision accepted June $26,1979$. 